\title{
Doppler ultrasound evaluation of pattern of venous incompetance and relation with skin changes in varicose vein patients
}

\author{
Pant HP1, Sharma $\mathrm{S}^{2}$, Bhattarai $\mathrm{S}^{1}$, Pandit SP${ }^{3}$, Maharjan $\mathrm{D}^{2}$ \\ ${ }^{1}$ Radiology resident, ${ }^{2}$ Consultant Radiologist, ${ }^{3}$ Associate Professor, Department of Radiology, NAMS, Bir \\ Hospital
}

\section{BACKGROUND}

Varicosity of lower limb is a common problem in agricultural country like Nepal. Doppler ultrasound is a standard investigation modality for confirmation of diagnosis, evaluation of severity and venous mapping to plan treatment. We aim to find out the epidemiology, common pattern of incompetance, severity assessment by doppler and assosciation of the important doppler variables with skin changes.

\section{METHODOLOGY}

Doppler venous study was done in 56 patients from june 2013 to july 2014. Patients with deep vein thrombosis and peripheral arterial disease were excluded. Altogether 56 patients, 96 limbs were studied. Data was entered in preformed proforma. Doppler study was conducted by senior radiologist with linear probes. Data was entered in SPSS software version 16 and statistical significance was calculated using chi square test.

\section{RESULTS}

Bilateral limb involvement was seen in 40 patients and unilateral in 16 patuents only. Skin related changes were seen in 58 limbs. Saphenofemoral junction incometance was seen in $79(82.2 \%)$ limbs and was most common pattern of incompetance. Overall, superficial veins were involved in $88(91.7 \%)$ limbs and deep in 30(31.2\%) limbs. Prolonged duration of varicosity $(>9$ year) $(p=0.000)$, bilateral limb involvement $(p=0.024)$, reflux in deep venous system $(p=0.002)$, larger Greater Saphenous Vein (GSV) size $(p=0.003)$, prolonged duration of reflux $(p=0.000)$ and perforator incompetance $(p=0.002)$ were associatedwith skin changes. GSV diameter more than $7 \mathrm{~mm}$ was associated with reflux significantly $(\mathrm{P}=0.002)$.

\section{CONCLUSION}

Superficial vein incompetance is common pattern compared to deep venous system. Sapheno Femoral Junction (SFJ) incompetance is the commonnest pattern. Larger caliber of vein, prolonged duration of reflux, reflux in deep venous system and perforator insufficiency are significanty associated with skin changes. 


\section{INTRODUCTION}

Varicose vein is a term for dilatation of superficial veins because of reflux in veins due to absent or incompetant valves, which occurs generally in lower limbs because of its dependant position. Venous reflux is defined in veins when the retrograde flow is present for more than 0.5 second. Varicose veins in long term lead to venous hypertension and skin changes; like edema, pigmentation and ulceration. This disease entity is termed as chronic venous insufficiency/disease(CVD). Chronic venous disease is very common problem affecting approximately $15 \%$ of men and $25 \%$ of women among general population as seen in Western studies $^{1}$. There is lack of literatures in our part defining its prevalence and pattern of reflux.

Varicosity and its skin complications are important cause of morbidity. Varicose veins can occur due to many causes which lead to incompetance in valve or pumping mechanism in lower limbs. Most of the causes are idiopathic, however in some, there may be history of deep vein thrombosis(DVT). Post thrombotic varicose vein is traditionally thought as cause of severe ulceration and lipodermosclerosis ${ }^{2}$. Varicose veins are reported more in females and in left limbs ${ }^{1,3}$ and they are more prevalent in pregnancy, older patients, obesity and few occuptional groups ${ }^{4}$.SFJ incompetance is the main cause of varicosity, though there are multiple possible sites of reflux including sapenopopliteal junction(SPJ), deep femoral and popliteal vein reflux, and perforators incompetance ${ }^{5}$. Color doppler and spectral study is gold stadard for evaluation of varicose veins $^{6}$. Doppler analysis is very important to locate the site of venous reflux, severity of reflux and venous anatomy which is important to plan surgical and even nonsurgical treatments like ablation ${ }^{3}$. On spectral analysis, longer duration of reflux and more number of perforators may be associated with more severe outcome ${ }^{3,7}$. We aim to find out epidemiology of varicose veins, pattern of varicosities, associated reflux sites, relation of size of saphenous vein and relux severity and any factors significantly associated with complications in these patients.

\section{MATERIALS AND METHODS}

All consecutive patients sent for venous doppler study in radiology department with clinical impression of varicose vein in lower limb from 1 July 2013 to 30 June 2014 were taken as study sample. Patients with superficial thrombophlebitis, deep vein thrombosis, peripheral arterial disease and pregnancy were excluded. Sixty one patient were followed, of them 5 were excluded because of associated peripheral vascular disease. Altogether, 56 patients(96 affected legs) were studied. Patient particulars were recorded in preformed performa and doppler was conducted by senior radiologist. Duplex ultrasound was done in Toshiba Aplio 400 machine with linear transducer,9 $\mathrm{MHz}$ frequency. B-mode, color doppler and spectral analysis was conducted in all patients. Study was done in standing position with weight transmitted in contralateral side while examining. Both limbs were evaluated when clinically indicated. Common femoral vein(CFV), proximal GSV, SFJ, superficial femoral vein(SFV), popliteal vein (PV), SPJ, proximal small saphenous vein (SSV), perforators were studied.

The superficial and deep veins were examined for patency, competence and size. The diameters of SFV and GSV were measured just distal to level of SFJ in the groin. Similar measurements of the popliteal vein and SSV were done in the popliteal fossa. Deep venous reflux was looked in the femoral vein in the groin, popliteal vein in popliteal fossa. Incompetence was also looked for at the junctional sites i.e. saphenofemoral junction (SFJ), sapheno-popliteal junctions (SPJ) and perforator veins. Incompetence at these sites 
were assessed during Valsalva maneuver and using color doppler and spectral Doppler analysis. Reflux was established if the duration of reverse flow exceeded 500 milliseconds in the superficial veins or 1,000 milliseconds in the deep veins, as recently recommended by Labropoulos et $\mathrm{al}^{8}$. Time duaration of reflux were also recorded. Perforators were looked for in well defined areas above knee and below knee in all sides. Perforators were specifically looked for in areas where varicosity is prominent and flow pattern/ reflux, and diameter of perforators were also noted. The number and distribution of the incompetent perforators were also recorded.

Data was entered in Statistical Package for Social Sciences(SPSS) version 16. Statistical significance was checked using chi square test.

\section{RESULTS}

Of the 56 patients, 40 patients had bilateral leg involvement. Altogether 96 legs were evaluated. Males comprised 30(53.6\%) cases and 26(46.4\%) cases were females. Mean age of presentation was 8.8(S.D=9.8) years. Significant number of patients were farmers and housewives. Commonest occupation of patients was farmer which was of 16 patients(28.6\%) in our study group(graph 1). Mean age of presentation after onset of problem was 8years(S.D.4.9).

Skin related chnages/Complications were noted in $58(60.4 \%)$ of limbs, pigmentation being the commonnest which was noted in 52 limbs. Six limbs had lipodermosclerosis and ulcearation only. Lipodermosclerosis was noted in 31 limbs. All patients with lipodermosclersis showed ankle edema. Only 3 patients had history of DVT. Isolated left leg was involved in 9 cases and isolated right leg was involved in 7 cases. Mean proximal GSV diameter in 96 legs was $8.58 \mathrm{~mm}(\mathrm{~S} . \mathrm{D} .=1.4)$. Mean proximal superficial femoral vein diameter and small saphenous vein diameter were $10.4 \mathrm{~mm}($ S.D. $=1.92)$ and $4.5 \mathrm{~mm}$ (S.D. $=0.95) \mathrm{mm}$ respectively (Table 1$)$.

Reflux was noted most commonly in SFJ. SFJ incompetance was noted in 79 limbs. Isolated SFJ incompetance was found in 48 cases $(50 \%)$ and combined deep venous reflux(DVR) and SFJ reflux was noted in $24 \mathrm{limbs}$. Isolated SFV incompetance was detected only in 4 limbs. Deep venous reflux was noted in 30 limbs, however isolated deep venous reflux when seen(4 limbs) was associated with perforator incompetance(Table 2). Overall, superficial reflux seen in $91.6 \%$ (88 limbs), deep reflux in 31.2\%(30 limbs) and Perforators reflux were detected in $63.5 \%$ (61 limbs). Mean duration of reflux in GSV, SFV and SSV were 1.52 secs, 1.7 secs and 1.98 secs respectively(Table 1).All these limbs with perforator showed perforators in calf. Mid calf was commonnest site(43 legs, $46 \%$ of cases) which was commonly associated with perforators in other areas(19 legs, $19.8 \%$ of cases) and thigh was least common which was seen in only (5 limbs,5.2\% cases)(Graph 2).

Almost all perforators were noted in posteromediaside, except 5 perforators noted in lateral side near ankle. Upper calf and thigh showed no lateral perforators. Isolated perforator insuffuciency was noted only in 4 limbs. Multiple perforators were noted mostly, mean number was 1.96(S.D.=1.13).

Skin changes were more seen when both lower limbs were involved with chi square value of 5.06 (P Value $=0.024)$. Gender didn't show any correlation with complications. Patient with history of illness for more than 9 years showed more complications $(\mathrm{P}=0.000)$ (Table 3). GSV diameter more than $9 \mathrm{~mm}$ was associated with more complications $(\mathrm{P}$ value $=0.029)$. With increasing duaration of reflux $(>2$ second $)$, GSV size also increased significantly $(\mathrm{P}=0.000)$. 
Pattern of reflux in superficial or deep veins also showed statistical correlation with complications $(\mathrm{P}=0.002)$, deep venous reflux showing more complications(Table 3). Also, perforator incompetant patients had more skin related complications $(\mathrm{P}=0.003)$. When 2 or more perforators were involved, the association with skin changes was not statistically significant, however it was significantly associated when 3 or more perforators were involved (Table 3). GSV size more than $7 \mathrm{~mm}$ was associated with SFJ incompetance $(\mathrm{P}=0.001)$ and more or equal to $10 \mathrm{~mm}$ was significantly associated with complications $(\mathrm{P}=0.003)$. SSV size more or equal to $5 \mathrm{~mm}$ is also strongly associated with SSV $\operatorname{reflux}(\mathrm{P}=0.000)$ (Table 4).

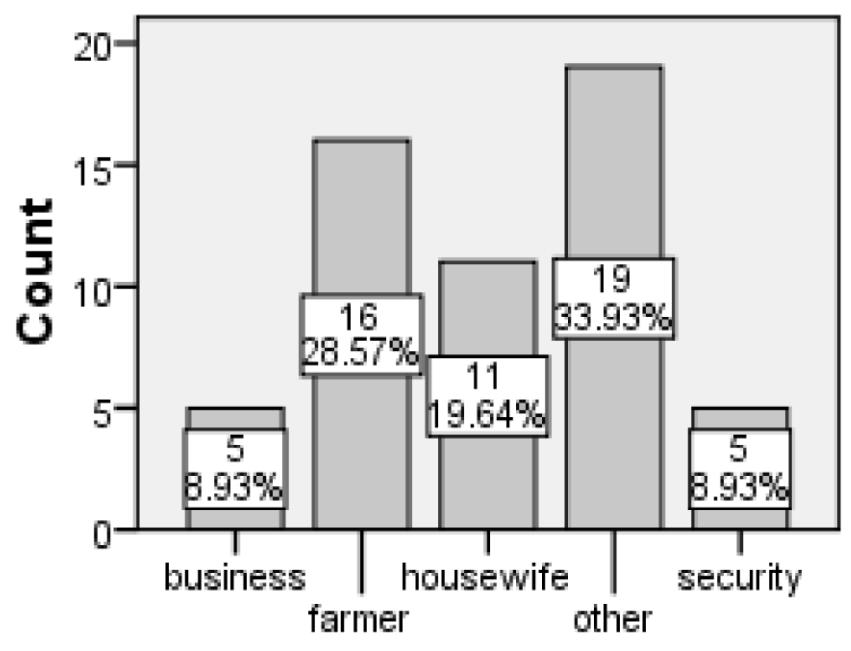

occupation

Figure1: Prevalence of different occupation in CVI patients

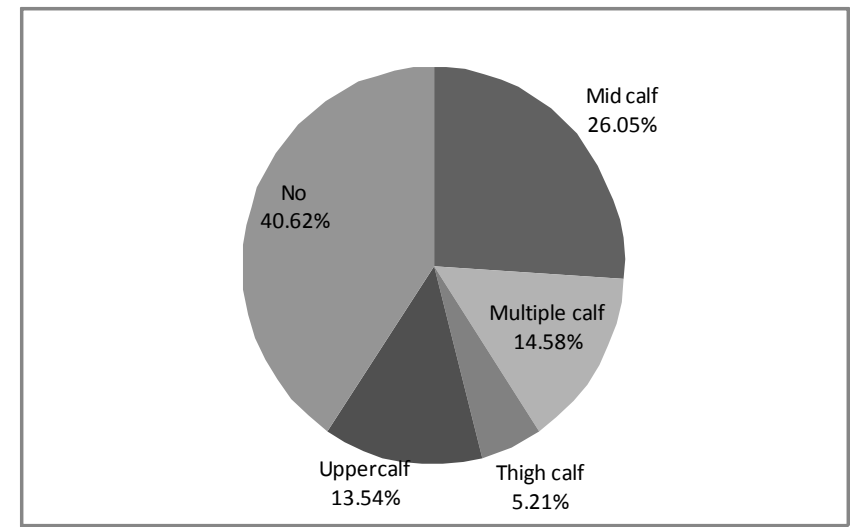

Figure 2: Prevalence of perforators and location in CVI patients

Table 1: Mean values of important doppler parameters

$\begin{array}{lll}\text { Parameters } & \text { Mean } & \text { S.D. } \\ \begin{array}{l}\text { GSV diameter } \\ \begin{array}{l}\text { Duaration of reflux } \\ \text { superficial vein }\end{array}\end{array} & 1.53 \mathrm{~mm} & 1.4 \\ \begin{array}{l}\text { SFV diameter } \\ \text { Duration of reflux }\end{array} & 10.4 \mathrm{~mm} & 0.6 \\ \begin{array}{l}\text { in deep vein } \\ \text { SSV diameter }\end{array} & 1.7 \mathrm{sec} & 0.67 \\ \begin{array}{l}\text { Duration of reflux } \\ \text { in perforators }\end{array} & 1.98 \mathrm{~mm} & 0.95 \\ \text { Number of } & & 0.6 \\ \text { perforators } & 1.96 & 1.13\end{array}$

Table 2: Pattern/site of reflux in CVI patients

$\begin{array}{lcc}\text { Incompetance site } & \text { Number } & \text { Percentage } \\ \text { Superficial system } & 88 & 91.7 \\ \text { Deep system } & 30 & 31.2 \\ \text { Perforator } & 61 & 63.5 \\ \text { Isolated GSV } & 48 & 50.0 \\ \text { GSV } & 79 & 82.2 \\ \text { SPJ } & 22 & 22.9 \\ \text { Isolated SPJ } & 7 & 7.2 \\ \text { Isolated SFV } & 4 & 4.1 \\ \text { Isolated perforator } & 4 & 4.2\end{array}$


Table 3: Statistical significance of epidemiological factors and doppler parameters on skin changes

$\begin{array}{lcc}\text { Parameters } & \begin{array}{c}\text { Chi square } \\ \text { value }\end{array} & \begin{array}{c}\text { P } \\ \text { value }\end{array} \\ \text { History of CVD }>9 \text { year } & 15.4 & 0.000 \\ \text { Female gender } & 3.5 & 0.058 \\ \text { Both limb involved } & 5.0 & 0.024 \\ \text { Reflux in deep system } & 9.58 & 0.002 \\ \text { GSV }>9 \text { mm } & 8.9 & 0.003 \\ \text { Duration of reflux }>2 \text { sec in GSV } & 18.2 & 0.000 \\ \text { Perforator incompetance } & 9.6 & 0.002 \\ \text { Perforator 2 or more } & 2.13 & 0.14\end{array}$

Table 4: Relationship of size of veins and reflux

$\begin{array}{lcc}\text { Parameters } & \begin{array}{c}\text { Chi square } \\ \text { value }\end{array} & \begin{array}{c}\text { P } \\ \text { value }\end{array} \\ \text { GSV }(>7 \mathrm{~mm}) & 10.5 & 0.001 \\ \mathrm{SFV}(>9 \mathrm{~mm}) & 33.9 & 0.000 \\ \mathrm{SSV}(>4 \mathrm{~mm}) & 17.9 & 0.000\end{array}$

\section{DISCUSSION}

Many professions as described elsewhere are risk factors for varicosity like farmers, security persons, so is obesity but we lack data regarding such epidemiological association in our part. Varicose vein was commonly found in farmers in our study probably because Nepal is an agricultural country and farmers work for prolonged hours in standing position(orthostasis). The no of males were more in our sample which is contradictory as varicosity is found more in females ${ }^{1,9}$, related to effects of hormones in females ${ }^{9,10}$. This may be explained by male seeking more health care as compared to females in our part.

Orthostatic position leads to constant venous pooling and damage to valves which leads to reflux in superficial and deep veins. Venous hypertension in superficial veins trasmitted through perforators during calf contraction in absence of competant valves can also lead to skin related changes. On the other hand, Post thrombotic syndrome generally leads to damage of valves in deep venous system and perforators and less in superficial venous system. But later on, superficial system also fails from the complex pathophysiology and CVD results. In our study, most cases were due to primary venous insufficiency.

Deep venous reflux was noted in 30 limbs(31.2\% of cases),however most of these cases showed associated superficial reflux and perforator insufficiency. These are consistent with previous findings ${ }^{11,12}$. All cases of post thrombotic reflux showed deep venous reflux. Most cases in previous studies also showed deep venous reflux in post thrombotic cases ${ }^{11}$. Saphenofemoral junction incompetance was most common pattern $(50 \%)$ which was also commonly associated with SPJ, SFV and perforator incompetance. Site of incompetance in our study was comparable to previous studies ${ }^{3,7,12,13}$. Perforator insufficiency ( $63.5 \%$ of cases) was mostly associated with incompetance in superficial and deep venous system, however isolated perforator insufficiency was seen in only $4.1 \%$ cases. Perforators incompetence can be the cause of varicose veinsbut themselves occur because of other reflux related to orthostasis, so overall isolated perforator incompetance is low, which was also found in our study. Perforators were mostly located in $\operatorname{mid}(46 \%$ of cases $)$ and upper calf( $28 \%$ of cases $)$. Perforators were mostly found in posteromedial aspect which is well established and described elsewhere ${ }^{3}$, but lower calfperforators were very less in our patients in contrast to some other studies ${ }^{3}$. Perforators incompetance leading to more severe skin changes has been documented elsewhere ${ }^{3,7}$ and we found the same. Generally multiple perforators( 3 or more) were noted in those with complications; however, 2 or more 
perforators were not associated with skin changes which was reported in some studies?

Diameter reflux relationship in superficial veins have been extensively studied ${ }^{14,15}$. GSV diameter more than $7 \mathrm{~mm}$ near SFJ is associated with reflux in most limbs ${ }^{13}$ which was also found in our study. We also found that SSV diameter more than $4 \mathrm{~mm}$ is associated with reflux, which supplements those studies. Bilateral leg involvement was associated with skin changes as compared to single leg involvement. This may be related to late presentation in patients with varicosity, presence of systemic disease/problems like obesity, family history of venous disease, chronic airway disease and higher age in those patients. Age as independent risk factor for severe disease has also been described ${ }^{16}$. We assessed different doppler parameters which may be associated with increased skin changes. Larger GSV, delayed presentation to hospital, presence of deep venous reflux, presence of perforators were associated with more skin changes. These were previously reported $^{3,7,8}$. We also found more skin related changes if there is increased duration of reflux which was not statistically significant in study by Irodi A etal ${ }^{3}$.

\section{CONCLUSION}

Bilateral venous involvement in common than unilateral venous involvement in varicose vein disease. Farmers are most common group to be affected. Superficial vein incompetance is common pattern as compared to deep vein incompetence. SFJ incompetance is commonnest pattern among junctional incompetance, though perforators incompetance is associated in most cases. Larger caliber of vein, prolonged duration of reflux, reflux in deep venous system and perforator insufficiency are significanty associated with skin changes.

\section{AKNOWLEDGEMENT}

We would like to thank surgery department for their academic help.

\section{REFERENCES}

1. Callam MJ. Epidemiology of varicose veins. The British journal of surgery. 1994;81(2):167-73.

2. Myers KA, Ziegenbein RW, Zeng GH, et al. Duplex ultrasonography scanning for chronic venous disease: patterns of venous reflux. Journal of vascular surgery. 1995;21(4):605-12.

3. Irodi A, Keshava SN, Agarwal S, et al. Ultrasound doppler evaluation of pattern of involvement of varicose veins in indian patients. Indian journal of surg. apr 2011;73(2):125-30

4. Beebe-Dimmer JL, Pfeifer JR, Engle JS, et al. The epidemiology of chronic venous insufficiency and varicose veins. Annals of epidemiology. 2005;15(3):175-84

5. Engelhorn CA, Engelhorn AL, Cassou MF, et al. Patterns of saphenous reflux in women with primary varicose veins. Journal of vascular surgery. 2005;41(4):645-51.

6. Dixon PM. Doppler ultrasound in the pre-operative assessment of varicose veins.Australas Radiol. 1996;40(4):416-21

7. Stuart WP, Adam DJ, Allan PL, et al.The relationship between the number, competence, and diameter of medial calf perforating veins and the clinical status in healthy subjects and patients with lower-limb venous disease. J Vasc Surg.2000 Jul;32(1):138-43 
8. Labropoulos N, Tiongson J, Pryor L, et al. Definition of venous reflux in lower-extremity veins. Journal of vascular surgery. 2003;38(4):793-98.

9. Cohen J. Venous insufficiency and oral contraception. Revue francaise de gynecologie et d'obstetrique. 1991;86(2 Pt 2):187-89.

10. Evans CJ, Fowkes FG. Prevalence of varicose veins and chronic venous insufficiency in men and women in the general population: Edinburgh Vein Study. J Epidemiol Community Health. 1999;53(3):149-53.

11. Ioannou CV, Giannoukas AD, Kostas T, et al. Patterns of venous reflux in limbs with venous ulcers. Implications for treatment. International angiology : a journal of the International Union of Angiology. 2003;22(2):182-87

12. Magnusson MB, Nelzen O, Risberg B, et al. A colour Doppler ultrasound study of venous reflux in patients with chronic leg ulcers. European journal of vascular and endovascular surgery : the official journal of the
European Society for Vascular Surgery. 2001;21(4):35360

13. Giannoukas AD, Tsetis D, Ioannou C, et al. Clinical presentation and anatomic distribution of chronic venous insufficiency of the lower limb in a typical Mediterranean population. International angiology : a journal of the International Union of Angiology. 2002;21(2):187-92

14. C Engelhorn, AEngelhorn, SX Salles-Cunha. Relationship between reflux and greater saphenous vein diameter. J VascTechnol, 1997;21: 167-72

15. FP Fonseca, AL Sarquis, SS Evangelista.Surgery for primary troncular varicose veins without stripping saphenous veins: Pre- and postoperative evaluation by duplex scan and photoplethysmography; Phlebology 1995 (Suppl 1), 419-21

16. Scott TE, LaMorte WW, Gorin DR, et al. Risk factors for chronic venous insufficiency: a dual case-control study. Journal of vascular surgery. 1995;22(5):622-28 